\title{
Anticholinergikum effektiv bei Mischharninkontinenz
}

- Die Prävalenz der Mischharninkontinenz ist bei Frauen aller Altersstufen höher als vielfach angenommen. Zu Beginn der Therapie empfehlen sich konservative Maßnahmen, gefolgt von lokaler Estrogenisierung und der Gabe eines Anticholinergikums.

Während die überaktive Blase (OAB) durch imperativen Harndrang, erhöhte Miktionsfrequenz sowie Nykturie charakterisiert ist und mit oder ohne Dranginkontinenz auftreten kann, ist die Belastungsinkontinenz durch unfreiwilligen Harnabgang beim Lachen, Husten oder bei körperlicher Anstrengung charakterisiert. Die Ursache der OAB ist eine unkontrollierte, spontane Detrusoraktivität, die der Belastungsinkontinenz eine insuffiziente Kontraktion des Schließmuskels oder des Beckenbodens, erklärte PD Dr. Christian Hampel, Mainz. Zur Basisdiagnostik gehört neben einer gynäkologischen und urologischen Anamnese die Erfassung der Hauptsymptome sowie des Leidensdrucks. Schon die allgemeine und die gynäkologische Untersuchung mit einfachen Funktionstests kann erste Hinweise liefern. Ergänzend nötig sind außerdem die Urinuntersuchung, die Restharnbestimmung und eventuell auch eine orientierende Sonografie, so Hampel.
Gelingt es mit konservativen Therapiemaßnahmen und einer lokalen Estrogenisierung nicht, die Harninkontinenz zu bessern, dann empfiehlt Hampel die Gabe eines Anticholinergikums. Bei dominanter Belastungskomponente kann zudem eine Inkontinenzoperation indiziert sein. Einer gegebenenfalls postoperativ in den Vordergrund rückenden Drangsymptomatik kann dann erneut mit einem Anticholinergikum begegnet werden.

\section{Neue Generation von Biopsiekanülen}

Die Stanzbiopsie-Kanüle Corazor ist das Ergebnis intensiver Forschungen, um in der Biopsie noch exaktere Ergebnisse zu erzielen. Durch eine Drei-Punkt-Fixierung wird die Kanüle exakt in ihrer Position gehalten. Ein Vibrieren beim Auslösen des Schussgeräts und damit ein Verfehlen des Zielgewebes sind dadurch so gut wie ausgeschlossen. Die Biopsie kann durch das Fachpersonal optimal vorbereitet werden, da der Kanülenschutz erst nach dem Einlegen der Kanüle in das Schussgerät und unmittelbar vor der Gewebeentnahme abgenommen werden muss. Darüber hinaus werden konstruktionsbedingt der direkte Kontakt der Kanüle mit dem Mehrfachschussgerät und damit die Kontaminationsgefahr verhindert. Der Luer-Anschluss an der Außenkanüle ermöglicht eine Aspiration oder die Anlage einer Lokal-Anästhesie. Mit dieser optimierten Diagnostik sind die Grundlagen geschaffen, ein Karzinom frühzeitig zu therapieren.

Nach Informationen von

Uromed, Oststeinbek

\section{Mit Grüntee-Extrakt gegen Genitalwarzen}

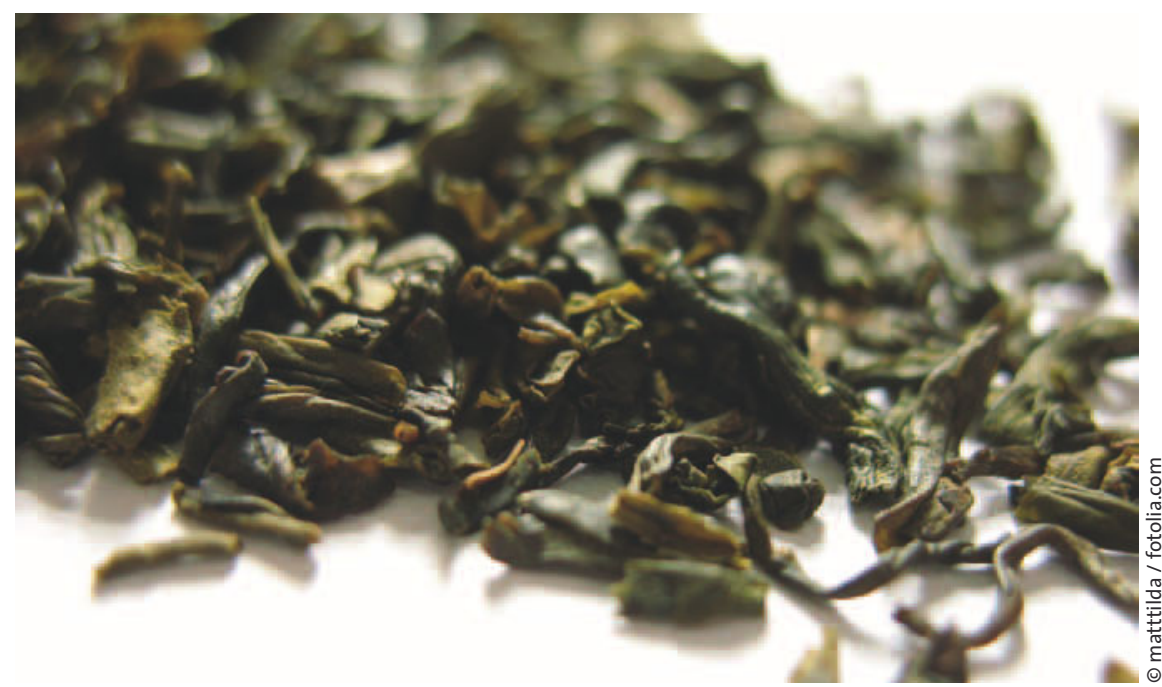

Mit Catechinen aus Grünem-Tee-Extrakt lassen sich Feigwarzen wirksam bekämpfen.

- Die Inzidenz von Genitalwarzen, die zu über $90 \%$ von den Typen 6 und 11 des humanen Papillomvirus (HPV) verursacht werden, steigt an. Zwar stehen bereits mehrere Behandlungsoptionen zur Verfügung, ein Problem der Therapie von Feigwarzen ist jedoch die teilweise hohe und variierende Rezidivrate. Durch die Einführung von Veregen ${ }^{\circledR} 10 \%$ Salbe hat sich das therapeutische Spektrum im letzten Jahr erweitert. Wirkstoff des neuen topischen Therapeutikums ist ein Trockenextrakt aus Grünem Tee mit Catechinen mit definierter Zusammensetzung. So hemmt Epigallocatechin- gallat unter anderem die Proliferation der mit den Viren infizierten Karatinozyten und beseitigt reaktive Sauerstoffmetabolite. Beeindruckend ist die in zwei randomisierten, placebokontrollierten, doppelblinden Studien dokumentierte Rezidivrate von $6,5 \%$ bei guter Verträglichkeit. Zwar können auch unter Veregen ${ }^{\circledR} 10 \%$ Salbe lokale Nebenwirkungen auftreten, diese sind jedoch minimal und führen erfahrungsgemäß nur selten zum Therapieabbruch.

Nach Informationen von

Abbott, Wiesbaden 